\title{
The development of the creative economy in Malaysia as experienced in two different case studies
}

\begin{abstract}
Cultural, tourism, and creative industries sectors can be understood as having intersecting policy agendas and economic interdependencies. In the developing context the first two have a somewhat longer history of analysis than the last one. However, emerging studies are beginning to emphasise all three domains are interconnected to each other, and need each other to sustain their products in the market. However, the studies are mainly concentrated on Western countries and there are still many aspects of this concept remain unanswered particularly in developing nations for example Malaysia. Data on developing nations is still problematic and considerably limited, or only concentrated in countries such as China and India. To have a better grasp about the underlying key concepts of the development issues on creativity, technology and innovation applied in cultural products in developing nations this study proposed case studies approach to be applied in Malaysia. The creative economy development issues in Malaysia will be the primary focus to be investigated by this research. An electronic research through the Internet on two different case studies in Malaysia had been looked at on their experience in developing their creative economy through a combination between culture, tourism and creativity.
\end{abstract}

Keyword: Component; Creative economy; Cultural tourism; Creative industries; Experience industries; Developing nations 